\title{
Experiencia de docencia basada en proyectos usando la música como elemento principal para la asignatura de Fundamentos de Electrónica
}

\author{
Quintián Pardo, Héctor ${ }^{1}$; Jove Pérez, Esteban²; \\ Casteleiro-Roca, Jose-Luis ${ }^{3}$; Calvo-Rolle, Jose Luis ${ }^{4}$ \\ ${ }^{1}$ Universidade da Coruña, Escuela Universitaria Politécnica, 0000-0002-0268-7999 \\ ${ }^{2}$ Universidade da Coruña, Escuela Universitaria Politécnica, 0000-0002-0625-359X \\ 3Universidade da Coruña, Escuela Universitaria Politécnica, 0000-0001-9740-6477 \\ ${ }^{4}$ Universidade da Coruña, Escuela Universitaria Politécnica, 0000-0002-2333-8405
}

\section{RESUMEN (ABSTRACT)}

La tendencia actual y futura en las carreras técnicas y en especial en las ingenierías, es que el número de alumnos se ve cada vez más reducido, bien por falta de vocación 0 motivación para afrontar una carrera si bien compleja, con una alta demanda laboral. Parte de esa falta de motivación proviene muchas veces de la necesidad de convertir muchos de los contenidos en temáticas más atractivas para los estudiantes. Por ello en esta experiencia de innovación docente, se ha planteado el uso de la música como principal elemento motivador para convertir el mismo contenido de asignaturas, en especial de los primeros cursos, en contenidos más atractivos a los estudiantes y conseguir de este modo, no solo un mayor efecto de motivación sino también que esto se refleje en los resultados finales obtenidos por los estudiantes. En este caso en concreto, se propone el desarrollo e implementación de un circuito electrónico capaz de "buscar" una canción "escondida" entre otras muchas, de modo que se incluye además un aspecto competitivo entre los alumnos. Tras la experiencia, los resultados obtenidos han sido muy positivos, tanto en el aspecto motivacional con un aumento de la participación de los estudiantes en un $22 \%$, así como en los resultados académicos obtenidos.

PALABRAS CLAVE: Música, motivación, electrónica. 


\section{CITA RECOMENDADA:}

Quintián Pardo, Héctor; Jove Pérez, Esteban; Casteleiro-Roca, Jose-Luis; Calvo-Rolle, Jose Luis (2020): Experiencia de docencia basada en proyectos usando la música como elemento principal para la asignatura de Fundamentos de Electrónica. En De la Torre Fernández, E. (ed.) (2020). Contextos universitarios transformadores: Boas prácticas no marco dos GID. IV Xornadas de Innovación Docente. Cufie. Universidade da Coruña. A Coruña (págs. 191-206). DOI capítulo: https://doi.org/10.17979/spudc.9788497497756.191 DOl libro: https://doi.org/10.17979/spudc.9788497497756

\section{ABSTRACT}

The current and future trend in technical careers and especially in engineering, is that the number of students is increasingly reduced, either due to lack of vocation or motivation to face a career although complex, with a high labor demand. Part of that lack of motivation often comes from the lack of converting many of the content into more attractive topics for students. For this reason, in this experience of teaching innovation, it has proposed the use of music as a main motivating element to convert the same content of subjects, especially the first courses, into more specific content to students and obtain in this way, not only a mayor motivational effect but also that this is reflected in the final students results. In this particular case, it is proposed the development and implementation of an electronic circuit capable of "searching" for a song "hidden" among many others, so that a competitive aspect among students is also included. After the experience, the results obtained have been very positive, both in the motivational aspect with an increase in student participation in more than $22 \%$, as well as in the academic results obtained.

\section{KEY WORDS: Music, motivation, electronic.}




\section{INTRODUCCIÓN}

Desde hace ya un tiempo la innovación educativa es un aspecto de vital importancia, independientemente de la disciplina en la que realice la formación (Ellis \& Childs, 2019). Sin embargo, dependiendo de la temática abordada, puede ser más o menos sencillo innovar en este sentido (Blouin et al., 2009). Si a lo mencionado se suma la importancia, el alcance y la implantación de las nuevas tecnologías en general, la innovación es una obligación (Wanta, 2003).

Obviamente las materias de ingeniería en general no son ajenas a esta necesidad imperiosa que existe (Aihua, Qiubo, \& Jie, 2019). De hecho, existe un problema en los países más avanzados con las vocaciones de los estudiantes, cuya tendencia actual es que los potenciales estudiantes rehúyen este tipo de estudios, los denominados STEM (Science, Technology, Engineering and Mathematics) (Dapozo, Greiner, \& Petris, 2016). Además, como problema añadido, es necesario mencionar que existe una gran brecha de género en las carreras que abarcan estos estudios (García-Holgado, Camacho, \&, García-Peñalbo, 2019).

En los últimos años se han hecho una serie de estudios preliminares en los que se trata de evaluar qué es lo que ocurre, una muestra de ello es la tesis doctoral (López-Vázquez, 2015). Además son numerosos los trabajos en los que se trata de valorar el rendimiento, como paso previo a la aplicación de técnicas de innovación, como muestra de ello se tienen los trabajos (López-Vázquez et al., 2015), (Crespo-Turrado et al., 2016), (Jove, Blanco-Rodríguez, et al., 2018), (Alaiz-Moretón et al., 2019), (Crespo-Turrado et al., 2020), (Jove et al., 2019), (Jove, et al., 2018).

Con el objetivo de paliar en cierta medida la problemática expuesta, en cuanto a la falta de motivación de los estudiantes por estudiar este tipo de carreras, con el fin de lograr motivar a los estudiantes que ya hayan escogido estos estudios, y además con el objetivo de motivar y mostrar la utilidad en una fase temprana de aquellos estudios que están realizando, se diseña un trabajo práctico, en el marco de la asignatura de Fundamentos de Electrónica de los grados 
de Ingeniería Eléctrica e Ingeniería Electrónica Industrial y Automática que se imparten en la Escuela Universitaria Politécnica de la Universidade da Coruña.

Para ello, se propone un reto en el que la música juega un papel principal, con objeto de generar una mayor motivación en el alumnado. Para ello, se les proporciona una canción mezclada con otras muchas de forma que esta sea inaudible y se propone a los alumnos crear un circuito electrónico que permita separarla de entre las demás. De este modo también se fomenta un aspecto competitivo entre los propios estudiantes, ya que deberán de competir entre ellos por ver quién consigue descubrir la canción buscada y con la mejor calidad posible.

\section{DESCRICIÓN DE LA EXPERIENCIA}

La experiencia docente propuesta se enmarca en la materia de Fundamentos de Electrónica (770G01018) de la EUP de la Universidade da Coruña. En dicha asignatura, al igual que en muchas otras en el ámbito de la Ingeniería Industrial, se detectaron los siguientes problemas:

- Falta de motivación y de interés de los estudiantes debido a:

- Los trabajos si bien están relacionados con la parte teórica no tienen una aplicación directa fuera del ámbito más técnico de la electrónica.

- Tasa de participación baja:

- A pesar de que el trabajo tiene una puntuación de hasta 2 puntos, al no ser de carácter obligatorio, muchos de los alumnos no se apuntaban al trabajo y otros no lo llegaban a terminar.

Por todo ello se planteó se planteó un trabajo que incluía diseño, simulación y montaje de un circuito electrónico que resolvía un problema. En este caso, con el afán de fomentar la motivación y participación de los estudiantes se planteó cómo temática central "la música". A pesar de ser una de las primeras materias de electrónica del grado, se trató de hacer hincapié en la parte práctica, especialmente para los alumnos que tenían mayor dificultad con los conceptos teóricos. 
Se pedía diseñar, simular e implementar un sistema de filtrado de audio que permitía separar una canción de entre varias. Para ello se les proporcionaba a los alumnos las siguientes herramientas:

- Programa de simulación de versión gratuita

- Audios premezclados

- Protoboard y componentes electrónicos necesarios

Esto permitía un trabajo más autónomo además de semi-presencial, ya que podían emplear su propio ordenador personal, tanto para escuchar los audios premezclados (varias canciones sonando el mismo tiempo), como los audios una vez filtrados (después de pasar por los filtros, el resultado podía ser introducido por la entrada del micrófono del ordenador y escuchar la única canción buscada filtrada).

\subsection{OBJETIVOS Y METODOLOGÍA}

De esta forma, se trataba de alcanzar los siguientes objetivos didácticos:

- Ahondar en los aspectos de funcionamiento de componentes electrónicos, en concreto los filtros de audio.

- Aumentar su pro-actividad a cara a solucionar los problemas que surgen, porque pueden "escuchar" los resultados del circuito diseñado.

- Introducir a los alumnos en una temática de la electrónica como es el filtrado y el audio.

- Aumentar la motivación de los alumnos al utilizar la música como elemento central del trabajo.

- Disponer de un material semipresencial.

- Facilitar la comprensión de los conceptos teóricos previamente explicados.

La metodología aplicada para introducir esta actividad consistió en:

1. Realización de una sesión sobre filtrado de señales.

2. Realización de una sesión de simulación. 
3. Tutorías.

4. Presentación del trabajo de forma "competitiva". El criterio es la calidad del audio.

Esta metodología busca fomentar el trabajo autónomo del alumno, que debía pedir los componentes diseñados el profesor de la materia y realizar la implementación y pruebas.

Además, el uso de una herramienta como la "música", tiene un alto factor motivador para el trabajo personal, así como el aspecto competitivo de tener que comparar el audio obtenido con los de los compañeros.

Por último, al poder realizar el trabajo tanto de forma presencial, como no presencial se fomenta la autonomía personal del estudiante.

El trabajo se dividió en varias partes, en una primera parte, se introducían algunos conceptos relativos al sonido y el oído humano (que no están directamente relacionados con la asignatura), a continuación, se explicaban las instrucciones para la realización del trabajo, así como los criterios de puntuación incluyendo un aspecto competitivo y por último se presentaba el enunciado del problema a resolver.

\subsection{CONCEPTOS PREVIOS NECESARIOS}

Con objeto de facilitar la comprensión del trabajo propuesto y motivar al alumnado se incluyo en la descripción del trabajo a realizar una pequeña introducción en relación con el sonido y el oído humano:

"El oído humano es un órgano que transforma las ondas sonoras en impulsos eléctricos que son interpretados por el cerebro. Un oído sano y joven es sensible a ondas sonoras en un rango de frecuencias de $20 \mathrm{~Hz}$ a $20 \mathrm{KHz}$.

A su vez el espectro audible puede dividirse en 3 tonos principales:

- Tonos graves, comprenden el rango de $20 \mathrm{~Hz}$ a $300 \mathrm{~Hz}$.

- Tonos medios, comprenden el rango de $300 \mathrm{~Hz} \mathrm{a} \approx 2 \mathrm{KHz}$.

- Tonos agudos, comprenden el rango de $\approx 2 \mathrm{KHz}$ a $16 \mathrm{KHz}$. 
El objeto de este trabajo consiste en poder separar los tonos graves de los agudos de dos archivos de música que han sido previamente mezclados."

\subsection{INSTRUCCIONES PARA LA REALIZACIÓN DEL TRABAJO Y PUNTUACIÓN DEL MISMO}

A continuación, se describía el trabajo a realizar indicando así mismo los aspectos que serían valorables, así como el sistema de puntuación, incluyendo el aspecto competitivo del mismo:

"El trabajo a realizar se dividirá en 3 secciones principales y consecutivas:

- Diseño: Se determinará mediante un diagrama de bloques las distintas etapas 0 bloques que tendrá el producto final en base a las especificaciones del trabajo. Será necesario diseñar y calcular todos los componentes necesarios para su posterior implementación teniendo en cuenta los componentes comerciales disponibles (ver listado de material disponible en el anexo).

- Simulación: Se simulará mediante Orcad la solución diseñada en el apartado anterior, en la medida de lo posible se usarán diversas páginas de simulación para obtener un proyecto estructurado. Será necesario que en la simulación se usen los mismos componentes con los que se implemente el circuito físico.

- Montaje: Se realizarán montaje en laboratorio sobre "protoboard" del circuito diseñado y simulado previamente.

Es imprescindible que cada etapa anterior funcione correctamente para pasar a la siguiente (diseño, simulación y montaje).

Puntuación: El trabajo valdrá 2 puntos sobre el total de la nota de la asignatura (ver presentación de la asignatura). Habrá un punto extra que se adjudicará de forma competitiva como resultado de las pruebas realizadas entre todos los circuitos presentados; para ello se realizará un ranking al final de la fase de pruebas (por votación entre participantes, sin posibilidad de auto voto) y la puntuación se asignará según la ecuación 1: 
Puntuación competitiva $=\frac{1 \text { punto }}{T T} *(T T+1-$ posición $)$

Donde $\pi$ el número total de trabajo presentados funcionales.

De modo que el primer trabajo de un total de 10 tendrá una puntuación de 1 punto, y el 10 una puntuación de 0.1 puntos.

\section{MATERIAL A ENTREGAR:}

- Memoria: Documento PDF donde se reflejen las soluciones de diseño elegidas en base a las especificaciones, así como los diversos resultados de las simulaciones y una sección que indique los detalles del montaje.

- Archivos de simulación: Se incluirá el proyecto de simulación completo.

- Implementación física: Se mostrará el funcionamiento el día de la presentación mediante prueba de audio del sistema de forma competitiva.

- PPT o similar que se usará el día de la presentación. LA DURACIÓN DE LA PRESENTACIÓN SERÁ DE 10 MINUTOS.

Contenido: Este será el principal punto a valorar. Se valorará tanto el correcto diseño como una adecuada y detallada simulación además de que el montaje funcione de forma correcta. La presentación de resultados por sí sola no se admitirá si no va acompañada de la correspondiente explicación de los mismos (figuras o tablas que no se expliquen no se valorarán).

Formato: El documento a entregar deberá tener una correcta edición y presentación (portada, índices, títulos numerados, tablas con pies de tabla, figuras con pies de figura, referencias, sin faltas de ortografía, etc.). Las imágenes deberán de ser de buena calidad y legibles. 
Implementación física: Se valorará una implementación clara con la menor cantidad de cableado, así como una clara distribución de los componentes sobre la "protoboard".

Presentación: Se valorará que se respete el tiempo de la presentación, el contacto visual con los asistentes, la presentación de conclusiones o de recursos visuales efectivos, entre otros.

En caso de hacer el trabajo entre 2 personas la nota es conjunta, por lo que ambos deben de tener el mismo conocimiento del trabajo realizado.

A tener muy en cuenta: Se va a emplear un software de detección de plagio con todos los documentos entregados. Aquellos que tengan un porcentaje elevado (aprox. superior a/ 30\%) se consideraran como suspensos."

\subsection{DEFINICIÓN DEL PROBLEMA A RESOLVER}

En este apartado se describe el problema a resolver por los estudiantes el cual está estrechamente relacionado con la música y el oído humano siendo la electrónica el hilo conductor principal, y usando los conceptos explicados en las sesiones de clase previas como elemento esencial para poder resolver el problema planteado. También se incluía un link con el material necesario, así como la descripción de los programas "gratuitos" necesarios.

"Se disponen de 2 líneas de transmisión de datos por la cuales llega música procedente de un ordenador (ver figura 1). Sin embargo, debido a un error de programación, el ordenador realiza la transmisión de música mezclando diversas tonalidades de varias canciones. Por ello, por la línea 1 se transmiten los graves de 1 canción con los agudos de otras canciones y por la línea 2 se transmiten los agudos de la primera canción con los graves de otras canciones (ver figura 2). 
Se pide entonces, diseñar un sistema analógico que permita obtener la canción buscada (canción 1), con todas sus tonalidades.

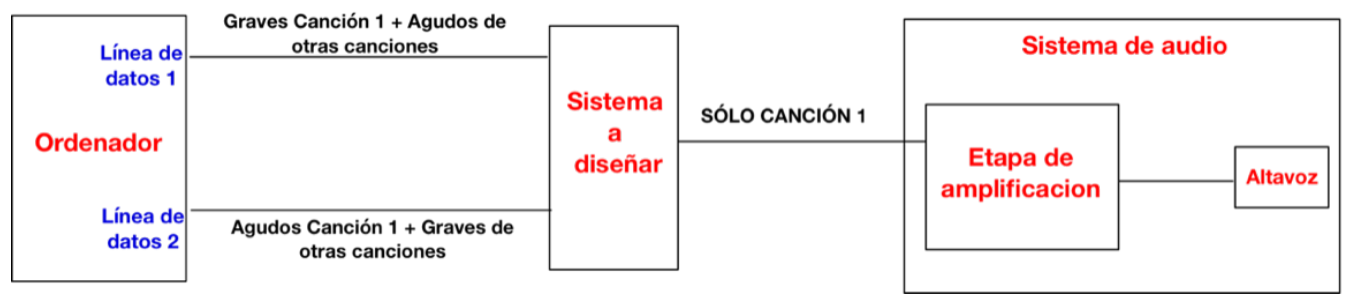

Figura 1. Esquema del sistema de transmisión de datos. Elaboración propia

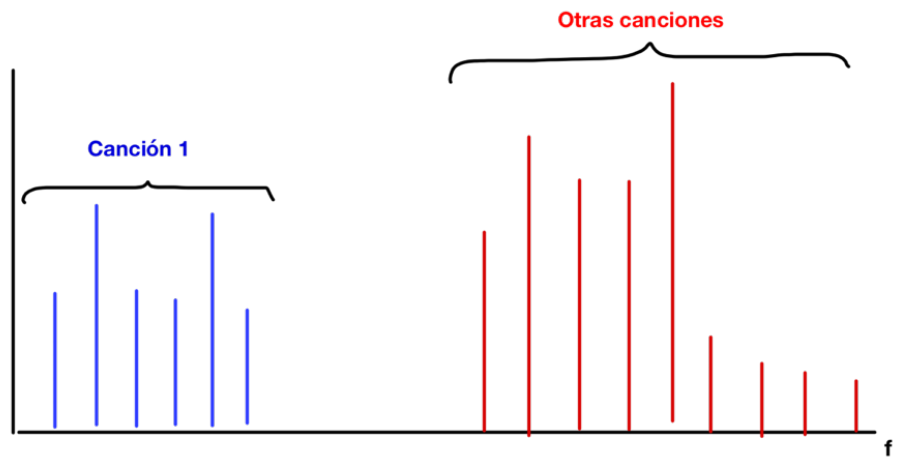

Línea de datos 1

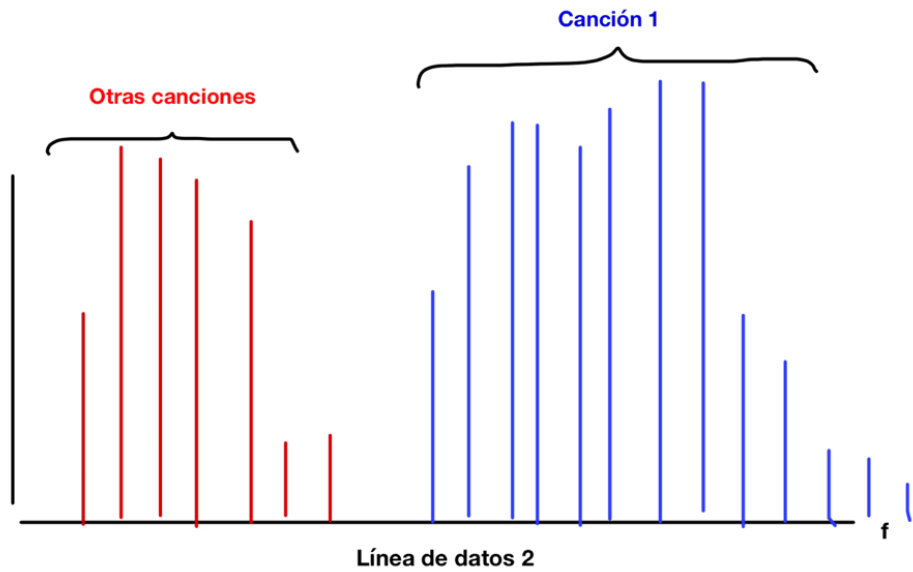

Figura 2. Espectro de frecuencia de los datos de la línea 1 y 2. Elaboración propia 
Por último, cabe mencionar que las señales de audio trabajan en un rango de tensiones de \pm 1 voltio, por lo que la salida del sistema no puede superar esos rangos de tensión ya que dicha salida irá a una etapa de amplificación de audio para su reproducción mediante altavoces (ver figura 1).

En el siguiente link (https://documenta.udc.es/share/s/CTDMjX04StWIYiRb7oyXIg) se dispone de una carpeta con todo el material necesario para el desarrollo de la misma, separado por carpetas:

- Edición y simulación: 2 archivos .txt uno para cada canal donde la primera columna es el tiempo y la segunda en nivel de tensión de la señal.

- canal1.txt

- canal2.txt

- Montaje: programa reproductor para generar el audio en las dos salidas (canal1 y canal2) y archivo de audio:

- reproductor.vi (programa LabView, instalado en las aulas)

○ audios.lvm

Por último, el sistema deberá cumplir las siguientes especificaciones:

- Los graves no deseados deberán de atenuarse al menos a la mitad del máximo armónico de los agudos buscados, sin que estos se atenúen más allá de $3 d B$.

- Los agudos no deseados deberán de atenuarse al menos 30db respecto al máximo de los agudos no deseados, sin que los graves deseados se atenúen más allá de $3 d B$.

- Deberá de disponerse de 2 controladores manuales que permitan reducir el volumen de los graves y los agudos de la señal final de forma independiente.

- El sistema diseñado deberá de testarse con señales de prueba del generador de funciones, antes de ser probado con los audios proporcionados." 


\section{RESULTADOS}

Tras la realización del trabajo por parte de los estudiantes, se procedió a realizar un cuestionario sencillo para evaluar los aspectos positivos y negativos de la experiencia. Además, se obtuvieron estadísticas relativas al índice participación, así como de resultados obtenidos, con objeto de valorar si el sistema propuesto había conseguido aumentar la motivación entre los estudiantes a la hora de abordar el trabajo, así como su efectividad en la asimilación de los conocimientos propios de la asignatura.

Figura 3. Índices de participación y tasas de abandono. Elaboración propia

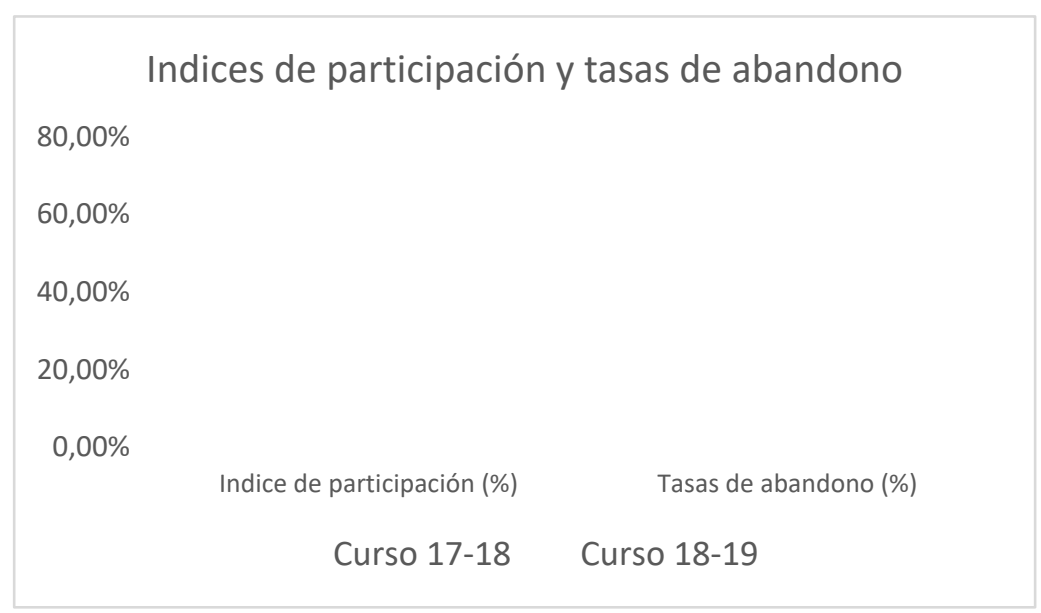

Figura 4. Índices de participación y tasas de abandono porcentuales. Elaboración propia 
Se realizó una encuesta de satisfacción entre los alumnos presentando los siguientes resultados (ver tabla 1):

\begin{tabular}{|l|c|}
\hline Preguntas & Media (0-7) \\
\hline Encuentro la aplicación práctica al contenido de la materia. & 5,50 \\
\hline Considero apropiada la realización del trabajo de forma semipresencial. & 4,48 \\
\hline $\begin{array}{l}\text { El trabajo realizado presenta una complejidad acorde con el temario impartido en las } \\
\text { sesiones de teoría y los problemas. }\end{array}$ & 3,52 \\
\hline $\begin{array}{l}\text { A partir del enunciado propuesto, he sido capaz de realizar distintas tareas de manera } \\
\text { autónoma, interiorizando conceptos básicos relacionados con la materia. }\end{array}$ & 5,61 \\
\hline Considero que el trabajo propuesto es interesante y me ha motivado a realizarlo. & 5,91 \\
\hline Estoy de acuerdo con la ponderación del trabajo respecto al total de la asignatura. & 4,00 \\
\hline
\end{tabular}

Tabla 1. Resultados de encuestas de satisfacción

Por último, en cuanto a las tasas de rendimiento académico, se obtuvieron los siguientes resultados:

- El $70 \%$ de los alumnos que realizaron el trabajo aprobaron finalmente la asignatura.

- De los alumnos que no realizaron el trabajo ninguno aprobó.

\section{CONCLUSIONES}

En base a la experiencia realizada y los resultados obtenidos, además del propio feedback con los alumnos, se puede concluir que el sistema propuesto tuvo una gran aceptación por parte del alumnado, ya que veían una aplicabilidad inmediata de aspectos teóricos de la electrónica, considerando muy positiva la experiencia. Todos consideraron que el factor que más les motivaba era el poder trabajar con música, y también el aspecto competitivo del trabajo era un gran aliciente. Muchos de los alumnos manifestaron su interés en este tipo de actividades en otras asignaturas. 
Desde un punto de vista más objetivo, en base a los resultados obtenidos, se obtuvieron varios objetivos:

- Aumento significativamente la tasa de ocupación de los laboratorios fuera de los horarios de docencia.

- Aumento objetivo del índice de participación:

- De un $45 \%$ a un $67 \%$

- Reducción de la tasa de abandono (si bien no era muy elevada):

- De un $16.6 \%$ a un $5.7 \%$

- El nivel de motivación conseguido es elevado.

- Se detecto que el esfuerzo requerido era medio-alto, por lo que la puntuación debía corregirse para compensar dicho esfuerzo.

Por lo tanto, la experiencia docente aplicada en este artículo ha sido de gran utilidad, permitiendo no sólo mejorar los resultados y motivación de los alumnos en una asignatura específica, sino que además las conclusiones y experiencias recogidas pueden ser exportadas y aplicadas en otras asignaturas con problemáticas similares.

\section{REFERENCIAS}

Aihua, W., Qiubo, Y., \& Jie, H. (2019). The Innovation and Reform of Higher Education under the Leadership of Emerging Enginering Education. Research in Higher Education of Engineering.

Alaiz-Moretón, H., Vázquez, J. A. L., Quintián, H., Casteleiro-Roca, J.-L., Jove, E., \& CalvoRolle, J. L. (2019). Prediction of Student Performance Through an Intelligent Hybrid Model. In H. Pérez García, L. Sánchez González, M. Castejón Limas, H. Quintián Pardo, \& E. Corchado Rodríguez (Eds.), Hybrid Artificial Intelligent Systems, pp. 710-721. 
Blouin, R. A., Riffee, W. H., Robinson, E. T., Beck, D. E., Green, C., Joyner, P. U., Persky, A. M., \& Pollack, G. M. (2009). Roles of innovation in education delivery. American Journal of Pharmaceutical Education. https://doi.org/10.5688/aj7308154

Crespo-Turrado, C., Casteleiro-Roca, J. L., Sánchez-Lasheras, F., López-Vázquez, J. A., de Cos Juez, F. J., Calvo-Rolle, J. L., \& Corchado, E. (2016). Student performance prediction applying missing data imputation in electrical engineering studies degree. Lecture Notes in Computer Science (Including Subseries Lecture Notes in Artificial Intelligence and Lecture Notes in Bioinformatics). https://doi.org/10.1007/978-3-31932034-2_11

Crespo-Turrado, C., Casteleiro-Roca, J. L., Sánchez-Lasheras, F., López-Vázquez, J. A., De Cos Juez, F. J., Pérez Castelo, F. J., Calvo-Rolle, J. L., \& Corchado, E. (2020). Comparative Study of Imputation Algorithms Applied to the Prediction of Student Performance. Logic Journal of the IGPL. https://doi.org/10.1093/jigpal/jzz071

Dapozo, G. N., Greiner, C. L., \& Petris, R. H. (2016). Estrategias innovadoras para favorecer el ingreso y la permanencia en carreras STEM. Extensionismo, Innovación y Transferencia Tecnológica. https://doi.org/10.30972/eitt.303024

Ellis, V., \& Childs, A. (2019). Innovation in teacher education: Collective creativity in the development of a teacher education internship. Teaching and Teacher Education, 77, 277-286. https://doi.org/10.1016/j.tate.2018.10.020

García-Holgado, A., Camacho Díaz, A., \& García-Peñalvo, F. J. (2019). La brecha de género en el sector STEM en América Latina: una propuesta europea. https://doi.org/10.26754/cinaic.2019.0143

Jove, E., Blanco-Rodríguez, P., Casteleiro-Roca, J.-L., Quintián, H., Moreno Arboleda, F. J., LóPez-Vázquez, J. A., Rodríguez-Gómez, B. A., Meizoso-López, M. D. C., Piñón-Pazos, A., De Cos Juez, F. J., Cho, S.-B., \& Calvo-Rolle, J. L. (2019). Missing data imputation over academic records of electrical engineering students. Logic Journal of the IGPL. https://doi.org/10.1093/jigpal/jzz056 
Jove, E., Blanco-Rodríguez, P., Casteleiro-Roca, J. L., Moreno-Arboleda, J., López-Vázquez, J. A., de Cos Juez, F. J., \& Calvo-Rolle, J. L. (2018). Attempts prediction by missing data imputation in engineering degree. Advances in Intelligent Systems and Computing. https://doi.org/10.1007/978-3-319-67180-2_16

Jove, E., López, J. A. V., Fernández-Ibáñez, I., Casteleiro-Roca, J. L., \& Calvo-Rolle, J. L. (2018). Hybrid intelligent system topredict the individual academic performance of engineering students. The International Journal of Engineering Education, 34(3), 895904.

López-Vázquez, J. A. (2015). Estudio de las materias y su correlación para innovación e implantación de las enseñanzas de ingeniería en una escuela técnica de Galicia.(Tesis doctoral). Recuperada de https://ruc.udc.es/dspace/bitstream/handle/2183/14849/ LopezVazquez_JoseAntonio_TD_2015.pdf

López-Vázquez, J. A., Orosa, J. A., Calvo-Rolle, J. L., de Cos Juez, F. J., Castelerio-Roca, J. L., \& Costa, A. M. (2015). A new way to improve subject selection in engineering degree studies. Advances in Intelligent Systems and Computing. https://doi.org/10.1007/9783-319-19713-5_47

Wanta, W. (2003). Preparing Graduate Students to Teach: Obligation and Practice. Journalism \& Mass Communication Educator. https://doi.org/10.1177/107769580305800302 